NOTAS Y DISCUSIONES

\title{
Rousseau político
}

\author{
EDUARDO BELLO \\ Universidad de Murcia
}

Podría tener razón o no J.J. Rousscau al afimar que utout tient à la politique». Lo cierto es que el problema político, tras la rememoración de la Revolución francesa $\mathrm{y}$, sobre todo, el resonar de la revolución democrática en los países del Este, contribuye hoy día a reafirmar la primacía de la razón práctica, que Kant formuló inspirado sin duda en Rousseau. Ahora bien, ¿qué quiere decir que todo radica o se anuda en la política? No es mi propósito contestar a la pregunta en estas páginas, proponiendo una interpretación más entre muchas, sino hacerme eco de las múltiples lecturas que de la filosofía política de Rousseau se han hecho en los treinta últimos años (1960-1990).

Es obvio que tal delimitación temporal es arbitraria. Con todo, no carece de justificación. Ante la imposibilidad de presentar aquí un nuevo repertorio bibliográfico, ${ }^{1}$ y dada la necesidad de adoptar algún criterio que permita racionalizar la selección de un abundante material, he creído conveniente adoptar como criterio cronológico el periodo más reciente de los estudios sobre Rousseau, a propósito de tres conmemoraciones muy significativas, al menos dos de ellas, desde el punto de vista que nos ocupa: el 200 aniversario de El Contrato social, la obra más representativa del pensamiento político del autor, en 1962; el aniversario de su muerte en 1978; y el de la Revolución francesa, en 1989. Estos acontecimientos histórico-teóricos han dado origen a tal producción -ediciones críticas, actas de coloquios, monografías, artículos y números especiales de revistasque resulta difícil establecer un criterio teórico de racionalización del cuantioso material. Como perspectivas de acceso a su estudio, nos guiaremos por los siguientes criterios: en primer lugar, nos ocuparemos en reseñar las aportaciones más relevantes en el periodo delimitado; en segundo lugar, y siguiendo un criterio teórico en sentido amplio, nos proponemos: 1) describir la recepción española en estos últimos años; 2) esbozar las interpretaciones ideológica y teóricamente más significativas; 3) finalmente, indicar los problemas que mayor debate o controversia ha suscitado la filosofia política de Rousseau, así como aquéllos que siguen teniendo mayor importancia en la actualidad.

\section{I}

De las tres efemérides indicadas, no cabe duda de que el bicentenario de El Contrato social tiene particular relevancia. Aunque 1962 no significa el 
descubrimiento del Rousseau político -Kant, Fichte, Hegel, Burke, Marx; E. Faguet, C.E. Vaughan, R. Derathé, entre otros, ya lo habían tomado en serio-, sí contribuye decisivamente a su consolidación como un clásico. Decisiva ha sido la edición crítica actualizada de los textos originales del autor; decisivas, las investigaciones suscitadas por ellos, por los nuevos tiempos y por el nuevo acontecer.

El volumen III de las Oeuvres complètes (Pléiade) de Rousseau lleva por subtítulo: Du contrat social. Écrits politiques. ${ }^{2}$ El primer sentimiento que experimenta un investigador al abrir este tomo - comenta M. Launay- es un sentimiento de gratitud. Más que los dos anteriores, «éste cra espcrado como una necesidad por todo el que quería estudiar las ideas políticas del siglo XVIII con un instrumento de trabajo seguro y cómodon. ${ }^{3}$ ¿Dónde encontrar, fuera de las bibliotecas, una buena edición de las polémicas que siguen a los Discursos, de las dos yersiones de El Contrato social, de las Lettres écrites de la Montagne y de los Escritos sobre la paz? ¿Cómo reunir los múltiples fragmentos políticos de Rousseau, publicados anteriormente con imprecisiones y de forma esporádica? Prologados y anotados por especialistas como J. Starobinski, J. Fabre - R. Derathé, los escritos políticos constituyen una herramienta indispensable no sólo para investigar el pensamiento político de Rousseau, sino también para comprender el desarrollo de éste desde la época moderna hasta nuestros días. Otro instrumento de trabajo de primer orden, complemento indíspensable de las Oeuvres complètes, es la edición criti- ca de la Correspondance complète de J.J. Rousseau, establecida y anotada por R.A. Leigh, profesor del Trinity College de Cambridge. ${ }^{4}$ De indudable interés teórico es la selección de cartas hecha por H. Gouhier, editadas con el título Lettres philosophiques. ${ }^{5}$ Entre otras, son célebres las dirigidas a Malesherbes (1762), en las que relata la iluminación de Vincennes que le hace ver "todas las contradicciones del sistema social», a Dom Deschamps (1761), a Mirabeau (1767), al señor de Saint Germain (1770). Según Starobinski, udeben ser leídas en pie de igualdad con los Discursos, el Contrato o las Confesiones».

De entre las investigaciones suscitadas por el bicentenario del Contrato, merecen especial atención las Actas de las Jornadas de estudio de Dijon y las del Coloquio de París. Las primeras, que han sido publicadas como Études sur le "Contrat social", 6 reúnen 32 colaboraciones ordenadas según tres bloques temáticos: las raíces del pensamiento político de Rousseau, aspectos de su pensamiento político en El Contrato social, el Contrato y el pensamiento político europeo desde 1762 a nuestros días.

Si el árbol gigante no carece de raíces profundas, a la teoría política de Rousseau no es difícil descibrírsclas en los pensadores modernos, sobre todo, porque él mismo confronta explícitamente con ellos su propio pensamiento. Así, Hobbes y Locke, Morelly y el abate de Saint Pierre constituyen algunos de los escasos nombres de la serie «raíces»; serie que aumenta considerablemente si extendemos la mirada a las monografías y artículos de revistas que tratan no sólo de teoría política, sino también de filosofia del derecho: Maquiavelo, Grocio, Pufendorf, Montesquieu, Diderot, Voltaire, Vico no agotan los nombres de fuentes o raíces. Asimismo la antigüedad romana ha sido objeto de una comunicación; pero sólo constituye un apunte de lo que desarrollará más tarde $D$. Leduc-Fayette en JJ. Rousseau et le my- 
the de l'antiquite. ${ }^{7}$ Es sabido que el Ciudadano de Gincbra cvoca figuras míticas como Sócrates, Catón, Licurgo, o ciudades míticas como Esparta y Roma. ¿Significa esta visión mítica de la antigüedad que Rousseau vive de espaldas a su tiempo, como se afirma con notable ligereza, con actitud regresiva? La Ciudad como espacio ideal de la República, entendida ésta como conquista incesante de la libertad y la igualdad, no constituye la manifestación de una actitud anacrónica, sino más bien anticipadora, nos dice Leduc-Fayette. O, como sostiene P. Casini, la referencia a la antigüedad mítica tiene una estructura bipolar: la imagen del pasado no es sino la base de un proyecto de futuro, esto es, el proyecto de la república perfecta, aunque tal futuro sea utópico. ${ }^{8}$

Tampoco el rastreo de fuentes agota la originalidad de Rousseau. Así lo subrayan los diferentes estudios sobre los problemas de la filosofía política en El Contrato social: la relación entre el Discurso sobre la desigualdad y el Contrato (J. Starobinski), entre el estado de naturaleza y la libertad; el sentido de la igualdad y de la desigualdad $(R$. Polin), lo social y lo político (P. Burguelin), la posición del problema de la política en Rousseau (S. Cotta); la Ciudad, el hombre, el ciudadano, la libertad y sus garantías; el papel del legislador, la palabra y la noción de gobierno; la religión civil, en fin, son algunos de los aspectos estudiados. ¿Problemas? S. Cotta no ha dudado en hablar de "totalitarismo democráticon," ${ }^{9}$ si bien casi todos los participantes han admitido, con C. Eisenmann, que la sociedad de El Contrato social no es la del Estado totalitario, sino la de la Ciudad democrática. ${ }^{10}$ Ahora bien, tan significativas como las cuestiones expuestas, han sido las silenciadas, por ejemplo, la voluntad general, la sobera- nía popular, el concepto de pucblo, el papel de la ley, la relación entre ética y política, el proyecto de paz, etc. Alguno de estos problemas ha sido el objeto de los estudios presentados en el Colloque International de Philosophie Politique (23-24 junio 1962) y publicados con el título Ronsseau et la philosophie politique. ${ }^{11} \mathrm{El}$ Coloquio ha hecho posible precisar aspectos del Rousseau político y confrontar interpretaciones ya conocidas como la tesis de la vía totalitaria (L.G. Grocker) refutada por la tesis de un Rousseau constitucionalista (E.F. Friedrich) basándose en la definición de República como un "Estado regido por leyes" (CS, II, 6).

De la tercera parte - la influencia del Contrato - cabe destacar, por una parte, los estudios que investigan la influencia en escritores políticos, por ejemplo, sobre Kant y Fichte (G. Vlachos) o sobre Marx (Della Volpe); por otra, la influencia sobre acontecimientos histórico-políticos, tales como las revoluciones de 1762 a 1789 (J. Godechot) o la Revolución francesa (A. Soboul); finalmente, la acogida hecha al Contrato en Suiza (C. Guyot) y su influencia en la cultura social. Como en los casos anteriores, tales estudios sólo constituyen una breve lista de la serie; así, por ejemplo, B. Bazcko extiende la influencia a Hegel, a Marx y a Freud, y A. Sánchez Vázquez, a Feuerbach, Engels y Kierkegaard, con especial referencia al acontecer de la independencia en México. ${ }^{12}$

Entre las monografías de este momento histórico, cabe destacar las de O. Vossler, I. Fetscher, B. Baczko, R.D. Masters. Vossler (1963), profesor de historia en Francfort, no escribe sin embargo un libro de historia. Centrando su análisis en El Contrato social (pp. 208-336), sostiene que los problemas políticos no constituyen el núcleo de las preocupaciones de Rousseau, 
sino sólo un aspecto de un sistema de pensamiento más amplio, en el que la idea de libertad - cuya evolución en la obra de Rousseau estudia - tiene la primacía. ${ }^{13}$ El libro de Fetscher (1960) es, probablemente, «la mejor obra escrita en lengua alemana sobre el pensamiento político de Rousseau", según M. Launay, Aunque el subtítulo remite a la tesis de Vossler -Para una historia del concepto democrático de libertad-, cabe observar al menos dos diferencias notables: una, que Fetscher escribe desde la perspectiva de la filosofía política, ya que en 1963 ocupa la cátedra de ciencias políticas en Francfort; la segunda, consecuencia de la anterior, es que presenta el estudio de la relación entre democracia y libertad mediante un análisis de los conceptos y problemas fundamentales del pensamiento político de Rousseau; considera Fetscher -tesis polémica- que el concepto central no es el de "contrato social» sino el de "voluntad general». ${ }^{14}$ La monografía de Baczko (1964), publicada inicialmente en polaco, pone de relieve el componente social de Rousseau político. No sólo la kalienación social» constituye uno de los cuatro capítulos de la obra, sino que la dialéctica individuo (soledad)/comunidad es el núcleo en torno al cual se articula el análisis de los demás problemas, tanto de las antínomias de la soledad, como de las aporías de la Ciudad; de ahí que "Libertad y utopía" sea el último problema planteado por el profesor de la Facultad de Letras de Ginebra. ${ }^{15}$ Entre las exposiciones más completas de la filosofía política de Rousseau, la de Masters ha de ser situada en primera fila. Metodológicamente, toma en serio la sugerencia de Jean-Jacques en Dialogtes, según la cual hay que comprender el sistema en su desarrollo lógico y unitario. Así, pues, Masters adopta como punto de vista los "principios» expuestos en el Emilio, y desde ellos analiza todos los textos y los problemas de la teoría política del ginebrino. De su investigación, bien documentada, podemos subrayar tres aspectos: no atribuye a Rousseau la paternidad de la "democracia totalitaria», como Talmon y Crocker; considera que no se puede aceptar la hipótesis del "pacto injuston; y el planteamiento del siguiente problema: themos de hablar de ruptura del iusnaturalismo -como sostienen L. Strauss y U. Cerroni- o de fines éticos - vinculados al derecho natural- de la Ciudad justa? ${ }^{16}$

De las tres efemérides evocadas al comienzo, la de 1978 - bicentenario de la muerte de J.J. Rousseau-va a quedar marcada por el giro social de la investigación de su filosofía política. La fuerza teórica y práctica de la izquierda en los años sesenta, que culmina en la explosión contestataria y reivindicativa de un cambio de valores -institucionales, políticos, culturales- ha forzado el giro en todo investigador sensible al acontecer de Mayo del 68. ¿Acaso Rousseau no es un predecesor de Marx en la crítica de la sociedad? G. Della Volpe, L. Colletti, B. Baczko, J.L. Lecercle, entre otros, serán leídos y traducidos por quienes buscan, frente al Rousseau romántico o totalitario, la nueva imagen de Rousseau teórico de la sociedad. ${ }^{17}$

Algunos trabajos, además de los mencionados, representan el espejo fiel del momento. Si J.N. Shklar estudia la teoría social de Rousseau y M. Berman lo presenta como precursor de las teorías sociales desarrolladas por $\mathrm{H}$. Marcuse y R.D. Laing, J. Charvet plantea lo que denomina "el problema social" de Rousseau: ¿ha descrito Rousseau de modo convincente - se pregunta- la transformación del hombre natural en hombre civil? ${ }^{18}$ Otros, sin dejar de ser 
representativos de la época, la traspasan por la profundidad del planteamiento de los problemas. Me refiero, sobre todo, a un libro de J. Starobinski y a dos monografías más, una de carácter colectivo, otra de J.G. Merquior. J.I. Rousseau. La transparencia y el obstáculo no es un libro de filosofía política; es mucho más. Está en la base, inspirändolas, de muchas de las obras reseñadas. Está en sintonía, auscultándolo, con el propio tiempo ya indicado. ¿Cómo remover el obstáculo, el mal que, a diferencia de la Teodicea de Leibniz, Rousseau adscribe a la sociedad? En el capítulo II, "La crítica de la sociedad", leemos: «cuando Engels estudie el Discturso sobre el origen de la desigualdad hará hincapié on el momento final del texto de Rousseau: los hombres sojuzgados, sometidos a la violencia brutal del déspota, recurren a su vez a la violencia para liberarse y para hacer caer al tiranon. 19 Obviamente, frente a la vía revolucionaria, en el Emilio se propone la vía pedagógica. ¿Se resuelve de este modo el problema social?

Un conjunto de estudios presentados en el Colloquium celebrado en Cambridge en $1978,{ }^{20}$ aborda de nuevo el problema bajo el epígrafe: «Política y sociología». Además de los estudios comparativos -Rousseau y Kant, Rousseau y Hume, Rousseau y Marx-. son significativos al respecto los presentados por B. Baczko ( sus lenguajes") y R. Derathe ( El lugar y la importancia de la noción de igualdad en la doctrina política de J.J. Rousseaun).

Insistiendo en el problema socio-político, Merquior abre un aspecto nuevo en esta línea de investigación. La novedad consiste ahora, por una parte, en comparar a Rousseau no con Marx, sino con Weber $y$, por otra, en plantear el estudio comparativo desde la perspectiva de la teoría de la legitimidad. La teoría de la legitimidad del poder (Rousseau) y la teoría de la legitimidad de la creencia (Weber) han de contribuir, según Merquior, no sólo a la dilucidación del concepto de «legitimidad", sino también a buscar una salida al problema político-social. ${ }^{21}$

En estrecha relación con dicha línea de investigación, se ha estudiado a lo largo de estos años la influencia de Rousseau en la Revolución francesa. De tal modo que el bicentenario de 1989 - aunque aún es prematuro afirmarlo- podría ser considerado, no tanto como un punto de partida, en el estudio de dicha proyección, sino más bien como un punto de inflexión. En tal inflexión confluyen dos o tres condiciones de nuestro tiempo. En primer lugar, ciertas lecturas de la Revolución francesa han estado condicionadas por la Revolución de Octubre, ${ }^{22}$ perspectiva que podría ser modificada $o$, al menos matizada, a partir de la revolución democrática en los países del Este. En segundo lugar, en el marco de estas coordenadas, el papel que juega la aportación de la tcoría de Rousseau tendría que ser revisado, para que salga a luz su transparencia frente a las criticas de uno y otro signo. Finalmente, tal revisión podrá hacerse más efectiva a partir de los textos de los oradores en las sesiones de la Asamblea Constituyente, que se editan en la actualidad, sobre todo en Gallimard.

Es cierto que alos 29 meses de la Asamblea Constituyente -observan F. Furet y R. Halévi-- son como un epilogo memorable a más de un siglo de interrogantes políticos y filosoficos sobre la sociedad moderna»; pero tal vez sea problemático afirmar a continuación que «se trata de un epílogo en el que intervienen condiciones no anticipadas ni previstas por ninguno de los pensadores ilustrados».23 Pues, sobre 
el papel que respectivamente han descmpeñado "la inteligencia" y las circunstancias ha escrito de forma sistemática D. Mornet. ${ }^{24}$ En un estudio reciente, R. Barny concluye que "si bien es evidente que el pensamiento de la Ilustración desempeña un papel capital, está claro que ese papel no es exclusivon. 25

Observamos, pues, que los distintos bicentenarios sobre la obra y vida de Rousseau han contribuido - al programar el estudio de su influencia - a investigar el papel capital de la Ilustración en la Revolución. ${ }^{26}$ Refiriéndonos en particular a la influencia de Rousseau, podríamos mencionar, a título de ejemplo, los trabajos de A. Soboul sobre el jacobinismo o sobre el concepto de soberanía popular y democracia directa entre las clases populares. ${ }^{27}$ Pero es necesario detenerse un momento ante tres importantes estudios: el libro bien documentado de McDonald, el excelente artículo de L. Sozzi, y el brillante ensayo de Starobinski. El primero recurre a documentos, libros, discursos, panfletos, periódicos -aunque limita su investigación al periodo 1789-1791-, si bien concluye que la interpretación conservadora es la que mejor ha respetado las tesis fundamentales del pensamiento político de Rousscau. Por su parte, L. Sozzi aporta las pruebas de un uso "reaccionario» de Rousseau más allá de 1792, es decir, de los monárquicos, de los aduladores de Bonaparte y de los defensores del cristianismo. Jean Starobinski, a quien debemos la reseña de estos dos trabajos, puntualiza en su ensayo, 1789, los embiemas de la razón, que "la prédica de Rousseau no ha "causado" la Revolución francesa, sino que ha incitado a los hombres de 1789 a comprender su situación como una crisis revolucionaria. El verbo de Rousseau - como el de los filósofos- sin haber determina- do el acontecimiento, ha suscitado el sentimiento que daba al acontecimiento su sentido majestuoso: ha desarrollado los conccptos que la reflexión y la acción política iban a poner a pruebaw, 28

¿Probarán lo contrario las nuevas investigaciones suscitadas por el bicentenario de 1989?

\section{II}

Además del punto de vista cronológico, seguido hasta aqui, señalábamos al comienzo algunos criterios teóricos que nos permitieran una mínima racionalización del abundante - ya desbordante- material, tales como la recepción en España del Rousseau político, las interpretaciones marcadas por el signo ideológico, así como los problemas de la filosofía política de Rousseau que mayor interés tienen para nuestra sociedad. El desarrollo breve de este esquema se lleva a cabo básicamente desde la doble perspectiva, temporal y teórica, que hemos seguido hasta aho$\mathrm{ra}$, es decir, ateniéndonos a las investigaciones del pensamiento político de Rousseau en las tres últimas décadas.

1. Por lo que respecta a la recepción del Rousseau político en España, la evolución de la sociedad ha impuesto su "tempus». Sin lugar a dudas, la transición y la Constitución de 1978 han sido más determinantes a la hora de interesarse por el fundador de la democracia moderna, que el bicentenario de la muerte de éste celebrado ese mismo año. Así, pues, resulta fácil observar la Presencia de Rousseau ${ }^{29}$ en las librerías y bibliotecas españolas bajo una triple perspectiva: a través de traducciones de monografias extranjeras -la tarea más rápida ante una necesidad inmediata y ante la carencia de un humus cultural en la época anterior, donde echara raíces la semilla de 
este tipo de investigación-, por medio de ediciones de los textos originales $\mathrm{y}$, paralelamente, de los primeros estudios sobre aspectos del Rousseau político.

Entre las obras traducidas, algunas son elocuentemente significativas no sólo por el autor, sino por la tesis que expresa el título en un momento clave de transformación de nuestra sociedad: L. Colletti, Ideologia y sociedad (1975), J. Moreau, Rousseau y la fundamentación de la democracia (1978); un poco más tarde se traduce la ya citada de J. Starobinski, J.J. Rousseau. La transparencia y el obstáculo (1983); y unos años antes, la de G. Della Volpe, Rousseau y Marx (1969). ${ }^{30}$ Sin pretender ser exhaustivos es preciso añadir que la serie de traducciones se ha ido enriqueciendo con algunas aportaciones más, como las de R. Grimsley (1977), B. Groethuysen (1985) y G. Sartori (1988). De indudable interés todas ellas, tienen en común que no estudian solamente el problema político en Rousseau. Sartori, en Teoria de la democracia, sólo le dedica unas páginas del volumen II, en el que estudia los «problemas clásicos»; Grimsley ofrece una excelente exposición de síntesis del pensamiento de Rousseau; mientras Groethuysen orienta más la sintesis hacia el Rousseau político y su influencia en la Revolución francesa. ${ }^{31}$

Pese a que la tarea de la traducción resulta insuficiente, cuando se trata dc leer a un pensador, dicha tarea se ha ampliado en este caso a la edición en castellano de los textos de Rousseau. Si la mediación de la monografía es un instrumento indispensable, aunque no suficiente, la lectura directa de un autor es la condición sine qua non para su comprensión. La lectura, en el idioma original, se dirá; cierto. Pero la lectura en el propio idioma también constituye una herramienta valiosa en la tarea investigadora.
Pues bien, a falta de una edición de las obras completas de Rousseau en castellano, algunas editoriales están realizando un gran esfuerzo en la edición de las obras fundamentales del Ciudadano de Ginebra. Desde la perspectiva de la filosofía política, cabe reseñar las siguientes. Además de los Escritos de combate (Alfaguara, 1979) y Del Contrato social. Discursos (Alianza, 1980), ${ }^{32}$ Editorial Tecnos nos ofrece dos novedades importantes: una, la edición por primera vez en castellano, del Discurso sobre la Economía politica (1985) y del Proyecto de Constitución para Córcega (y) Consideraciones sobre el gobienno de Polonia (1988), además de los Discursos (1987) y El Contrato social (1988); la otra consiste en la aportación metodológica de estas ediciones, a saber, la presentación del texto original anotado y precedido de un estudio preliminar, que facilita la comprensión del texto, así como de una selección bibliográlica para información del investigador o del lector, ${ }^{33}$ También es novedad la edición de Escritos sobre la paz y la guerra (1982); y no podemos olvidar las diferentes ediciones del Emilio, no sólo porque el Libro IV contiene una importante sintesis de teoría social ${ }^{34}$

El resultado de la investigación, publicada luego en monografías y otros estudios, creo que no ha hecho sino comenzar; si bien ha comenzado con buen pie, a juzgar por los libros de M.J. Villaverde y de J.L. Hernández. En el primero, Rousseau y el pensamiento de las Luces, la autora nos presenta un Rousseau conservador en tres tiempos: 1) enmarcado en la corriente tradicionalista y conservadora del siglo $X V I I, 2)$ empeñado en la crítica de la propiedad privada como crítica anticapitalista y en el mito del igualitarismo agrario, 3) defendiendo un modelo político anacrónico. Si alguna moderni- 
dad hay en Jean-Jacques, afirma la autora, ues esencialmente fruto del fracaso de su sistemas (p. 258); y wen el terreno político, el aspecto más innovador de su doctrina, el concepto de soberania popular, se fundamenta, como ha señalado Derathé, en los teóricos del Derecho natural" (p. 259). Tesis polémica, sin duda alguna - pero bien documentada-, para quienes opina* mos que Rousseau es, con Kant, el pensador ilustrado más lúcido, el predecesor de Marx al pretender erradicar los males sociales trabajando sobre los conceptos de "propiedad», «igualdad», "hombre", «Estado", es decir, sobre la dimensión moral y antropológica de la economía y de la política; el fundador (utópico), en fin, de la democracia moderna ${ }^{35}$ En La ley del corazón, J. Lópcz Hernández escribe: «El objetivo principal de este estudio era, en principio, la comprensión de la teoría política de Jean-Jacques como elemento configurador de la conciencia política contemporánea». (p. 16). Ahora bien, dado que en Rousseau, lo político, lo social y lo antropológico son interdependientes, la monografía -escrita con estilo ágil y bien documentadase ha centrado en el análisis de los tres niveles indicados, sin haber agotado el estudio de las diferentes dimensiones de lo político. Su tesis puede resumirse así: no podemos hablar del ciudadano, si no nos preguntamos antes acerca de lo que es el hombre; sin una base antropológica es muy difícil construir la legítima sociedad política. ${ }^{36}$

Además de otras monografías, como las de Caballero Harriet, Ginzo y Dalmaso, ${ }^{37}$ son valiosos los trabajos publicados en el número especial de la Revista de Estudios politicos (8, 1979): I. Fetscher, "La filosofía moral y política en Rousseau»; M.C. Iglesias, $₫ \mathrm{El} \mathrm{Pa-}$ raíso perdido en las Cartas persas y en los discursos rousseaunianos»; D. Ne- gro Pavón, "Rousseau y los orígenes de la política de consensom; J.M. Ripalda, "Hegel y Rousseau». Problemas de interés publicados en la misma revista o en otras publicaciones son: la interpretación kantiana del contrato social, los límites de la democracia directa, la división de poderes y la filosofía de la historia, entre otros. ${ }^{38}$

Cabe afirmar, pues, que la investigación sobre la filosofía política de Rousseau en España, aunque acaba de empezar, no puede haber comenzado con mejor pie, apoyando teóricamente el actual Estado democrático de Derecho.

2. En la primera página de El Contrato social nos advierte Rousseau que «si fuese un príncipe o un legislador no perdería el tiempo diciendo lo que hay que hacer". Pero como no es ni una cosa ni otra, ha dedicado discursos, cartas, ensayos y tratados a decir alo que hay que hacer", para encontrar «una forma de administración legítiman. Ahora bien, es frecuente que los diferentes lectores de esos textos no estén de acuerdo a la hora de precisar lo que ha dicho acerca de lo que hay que hacer. No están de acuerdo, sobre todo, porque cada cual confronta lo que ha dicho con su propia ideología; cada uno busca en la teoría del Ciudadano de Ginebra un aval o un oponente de su propia opción política; cada cual se propone encontrar en el discurso rousseauniano un argumento para sus propias representaciones (ideas, conceptos, a veces mitos) de lo que debe ser la sociedad y la política. De ahí las múltiples interpretaciones. De ahí el conflicto entre las diferentes lecturas. De ahí cl aspecto proteico de alguna que otra exégesis. Cabe observar, entre otras, las siguientes interpretaciones del Rousseau político: la conservadora, la liberal y la marxista, sin entrar en la posibilidad de la neutralmente académica. Aun así, hay que tener en cuenta 
que, como en la vida real, la uniformidad de las formas puras es infrecuente.

Pues bien, para la interpretación conservadora, Rousseau o bien es el destructor del orden social, o bien el que proporciona la teoria de la manipulación a los regímenes totalitarios del siglo $\mathrm{xx}$. Los primeros -J. de Maistre, De Bonald, Chateaubriand, por ejemplo-, consideran que el "orden social» que hay que preservar es el garantizado por la monarquía absoluta, cuyo poder es de origen divino, es decir, el sistema que aleja al pueblo de toda participación en el poder, esto es, la sociedad del Ancien Régime; por ello la noción de contrato social, que abre a tal participación, no es sino un sofisma, una máquina de destrucción ${ }^{39}$ que hay que aniquilar a cualquier precio. No en vano el conde De Maistre constituye una de las fuentes de inspiración del tradicionalismo religioso y político. En esta línea hay que situar el segundo tipo de argumentos que esgrimen Talmon y Crocker. Este último, en Rousseau's "Social contract". An interpretive Essay (1968), atribuye a Rousseau la patcrnidad del totalitarismo, basándose en la figura del legislador de la Ciudad y en el papel del preceptor de Emilio, cuya acción formadora somete a los hombres al "yugo" de la razón y les impone el orden que elegirán libremente... ${ }^{40}$ Según Starobinski, a esta lectura "maximalista" del Rousseau político —que acentúa con pesadez los elementos autoritarios represivos, por ejemplo la voluntad general desvinculada de su raiz consensual- se contrapone una interpretación umínima», según la cual no hay sociedad sin un mínimo acuerdo general de los individuos, para alejarse del reino de la violencia y entrar en el del derecho que emana de la norma consensuada.

La interpretación liberal, apoyada en la crítica que del contrato social han hecho B. Constant y Tocqueville, ${ }^{41}$ ve en la teoría de Rousseau un obstáculo a la libertad individual, a la defensa de los intereses privados y, en fin, al derecho inalienable a la propiedad, que ya formuló Locke. De ahí que la crítica liberal unas veces se une a la conservadora para atribuir a Rousscau la paternidad de la "democracia totalitaria" (L.J. Halle), y otras prefiere verle como un anacrónico que vive de espaldas a su tiempo (M.J. Villaverde) o, lo que es más frecuente, se le compara con otro teórico de mayor solera liberal, como Locke y Kant, pero también con Hobbes, Hegel o Burke, por ejemplo. ${ }^{42}$ Pero no es infrecuente que la lectura liberal haga del pensador de la libertad y del teórico de la democracia uno de los suyos, contribuyendo al mismo tiempo a refutar la acusación de predecesor de los totalitarismos del siglo Xx. En efecto, Chapman (Rousseau, totalitarian or liberal?) no sólo rechaza tal acusación sino que muestra una cierta aproximación -a pesar de notables diferencias- entre el pensamiento político de Rousseau y el liberalismo clásico, apoyándose para ello en los conceptos de voluntad general (Rousseau) y de Estado deliberativo (Lindsay) y en la noción clave de autonomía individual. ${ }^{43}$

Finalmente, si según Pasqualucci Rousseau nos lleva más directamente a Marx que a Kant, no es difícil comprender que la interpretacion marxista se haya interesado, a pesar de las variaciones de ésta, en el sentido de la filosofía política de Rousseau. Desde luego, es superficial plantearse si es el fundador del comunismo moderno $\left(\mathrm{Ja}_{\mathrm{a}}\right.$ net) o si su comunismo es pura apariencia (Kautsky). Tal controversia ${ }^{44}$ no hace sino ocultar el verdadero problema, a cuya solución contribuye la teoría sociopolítica de Rousseau. A dicho ocultamiento han cooperado trabajos 
como los de Althusser, el cual -poniếndose la lupa del Círculo epistemológico- no ve en El Contrato social sino deslizamientos, rupturas y contradicciones ${ }^{45}$ y A. Illuminati, para quien Rousseau no es sino el fundador de los valores burgueses. ${ }^{46}$

Entre las investigaciones que han descubierto, en cambio, el verdadero problema, yendo al fondo de la cuestión, hay que situar en lugar preferente las de Della Volpe, Colletti, Baczko y Lecercle. Para el autor de Rousseat y Marx es superficial considerar a Rousseau como filósofo utópico de los upequeños Estados", pues «la esencia fecunda del mensaje rousseauniano sobre la libertad (igualitaria) debe verse en la instancia universal (democrática) del "mérito" personal, o sea, en la exigencia del reconocimiento (social) de todo individuo humanon. ${ }^{47}$ No otro es el mensaje que también ha comprendido Colletti, para quien Rousseau no sólo es el crítico de la desigualdad social, sino sobre todo el teórico que, mediante el concepto de soberanía popular, ha establecido las bases programáticas de la revolución permanente ${ }^{48}$ o transformación incesante, en versión actualizada. El reconocimiento (social) de todo individuo, la crítica de la sociedad desigual no son sino dos aspectos del mismo problema, a saber, el problema que se plantean sin cesar las sociedades modemas: el problema de las relaciones entre el individuo y la comunidad, tal como lo evidencian las monografías de Baczko y Lecercle. ${ }^{49}$

No cabe duda de que el conflicto de las interpretaciones responde a supuestos cuya complejidad excede el mero trabajo académico. No cabe duda de que la complejidad no sólo radica en el texto, sino también en el lector, que no es un intelectual sin raíces sociales. No cabe duda de que tanto en el texto como en el lector resuenan, de un modo u otro, una vida, una época, toda una orquestación, en fin, de problemas sociales.

3. Sería lamentable que, en las diferentes interpretaciones, se nos ocultara precisamente aquello mismo que deseamos saber: el problema fundamental que plantea Rousseau. ${ }^{50} \mathrm{La}$ virtualidad de la lectura conservadora consiste en que nos permite ver el blanco contra el cual disparan una y otra vez: los conceptos de contrato social, voluntad general y soberanía popular, entre otros. $Y$ si hoy sabemos que la aportación liberal constituye uuna conquista histórica irreversibles, 51 en cuanto a la lucha por las libertades y por el Estado de Derecho se refiere, no hay que olvidar que Rousseau ha contribuido a tal conquista, no menos que Locke, Montesquieu, Pufendorf o Kant. Tal vez por ello, un marxista como Lecercle titula su ensayo: J.J. Rousseau, la modernidad de un clásico. Si bien no puede tomarse a un clásico al pie de la letra, se le reconoce porque los nuevos hechos no caen nunca fuera de su competencia.

En cfecto, cuando Bobbio plantea el problema actual de la crisis de la democracia, recurre para su tratamiento a la lección de los clásicos. Rousseau no sólo vio las dificultades de la democracia ideal - sólo posible sí fuéramos dioses-y las de la participación directa ${ }_{1}$ sino que criticó duramente la democracia representativa..$^{52}$ Acaso por haber planteado correctamente el problema, le reconoce Della Volpe el título de "padre de la democracia moderna»?

L. Colletti añade algo más. Considera, basándose en Gierke, que Rousseau ha resuelto el problema de la distinción entre soberano y gobiemo, formulando además el principio de la primacía de la soberanía popular, clave de la revolución permanente $o$, en lenguaje de hoy, transformación incesante. De 
ahí su tesis según la cual la teoría «política" revolucionaria, tal como se ha venido desarrollando desde Rousseau, está ya prefigurada y contenida en $E l$ Contrato social; o lo que es igual, para ser más explícitos, que, por cuanto concieme a la teoría "política» en sentido estricto, Marx y Lenin no han añadido nada a Rousseau, salvo el análisis (ciertamente muy importante) de las «bases economicas" ${ }^{53}$

¿Renace, por ello, la noción de contrato social, pese a que las críticas de historicistas, conservadores y positivistas se habían erigido en brazo ejecutor de la aniquilación de una noción que De Maistre calificaba de smáquina de destrucción»? Según Bobbio el nuevo interés por el contractualismo tiene una doble motivación: una circunstancial; la otra, vinculada a un problema actual. La circunstancia ha sido el exito del libro de Rawls, el cual parte precisamente de sla conocida teoría del contrato social tal como se encuentra, digamos, en Locke, Rousseau y Kants como supuesto de su teoría de la justicia. ${ }^{54} \mathrm{El}$ nuevo problema que hay que resolver, a nivel internacional según Bobbio, ya no es el del orden, sino el de la justicia. De ahí que sla propuesta de un nuevo pacto social global, y no parcial», constituya hoy una necesidad, a condición de sque sea introducida en el pacto alguna clausula que asegure una distribución equitativa de la riquezam, 55

La cláusula no es sino la expresión del principio ético de una mayor igualdad, principio insistentemente postulado por la exigencia de lo que se ha llamado "giro social». Con relación al derecho inviolable a la propiedad enunciado por Locke, Rousseau formuló en los siguientes términos el giro social: "... en cuanto a la riqueza - puntualiza aclarando su concepto de igualdad- que ningún ciudadano sea suficientemente opulento como para comprar a otro, ni ninguno tan pobre como para ser obligado a venderse» (CS, II, 11).

La ćtica de la igualdad se constituye, asi, en el supuesto indispensable al derecho a la libertad, esto es, de la autonomia individual (la libertad definida como obediencia a la ley que todos se han prescrito). No otro es lo que Bobbio llama el "argumento ético" de la mejor forma de gobierno, históricamente buscada. ${ }^{56}$

Cabe señalar, finalmente, que si el objetivo básico del Estado de Derecho consiste en la garantía de los derechos fundamentales de la persona humana, el argumento ético se constituye hoy en el criterio último de la legitimidad del Estado (democrático) de Derecho. ${ }^{57}$ Una contribución al debate sobre el concepto y los criterios de legitimidad es, sin auda, el libro de J,G. Merquior, Roussean and Weber. Two Studies in the Theory of Legitimacy. ${ }^{58}$ El autor contrapone dos perspectivas de un mismo problema: la teoría de la legitimidad del poder (power) y la teoría de la legitimidad de la creencia (belief. Pero tal contraposición de entrada no conduce a un distanciamiento de la posición de Weber respecto de la de Rousseau, sino a lo contrario: «La sociología histórica de Weber -concluye Merquior - constituye, por lo tanto, el sistema más accesible a la comprensión de la relevancia actual de la teoría de la legitinidad de Rousseau (p. 207).»

Todo un programa en cuya investigación aun estamos implicados si se prefiere -con Rousseau y Bobbio- el gobierno de las leyes al gobierno de los hombres, siempre que hayamos descubierto la legitimidad de las normas y hayamos optado por la mejor forma de gobierno. 


\section{NOTAS}

1. El mejor repertorio bibliográfico sobre J.I. Rousseau se encuentra en Annales Jean-Jacques Rousseau, 1904-1990, 40 vols. Cf también $R$. Trousson: Rousseau et sa fortune litteratre, París, Nizet, 1977; "Quince annés d'studes rousseauistes $*$ Dix-huitième siecte, 9 (1977). 343-386. J. Lopez Hernández: "Breve historia de la fortuna litemaria y de la crítica de J.J. Rousseau, Siglos xva y $\mathrm{xx} *$, Pensamiento, 162 (1985), 179-200; «... Siglo $\mathrm{xx}$ *, Pensamiento, 164 (1985), 431-452. Cf. la nueva revista: Etudes Jean-Jacques Rousseau, 1, Reirns, Ed, A. l'Écart, 1987.

2. J.J. Rousseau: Oeuvres completes, ed. de B. Gagnebin et M. Raymond, París, Gallimard (Pleiade), 1964, vol. III.

3. M. Launay: «Bbliographie Anstales I.7. Rousseau, t. XXXVT, 1963-1965, p. 406. Cf. The political Writings of Jean-Jacgues Roussenu, ed. by C.E. Vaughan, Oxford, B. Blackwel), 1962 (1st ed. 19.15).

4. J.J. Rousseau: Correspondence complète, Ed. de R.A. Leigh, Genève, Institut et Musée Voltaire, t. I et II, 1965; a partir del t. XXV, Oxford, The Voltaire Foundation at the Taylor Institution, t. XLIX (1989).

5. J.J. Rousseau: Lettres phitosophiques, ed. de H. Gouhier, Paris, J. Vrin, 1974.

6. Etudes sur le "Contrat social" de JJ. Rousseatt. Actes des Joumées d'Étude organisées à Dijon pour la commernoration du 200e anniversaire du aContrat social», París, Societé Les Belles Lettres, 1964.

7. Paris, J. Vrin, 1974. CF. A. Bruno: La formazione del pensiero politico de Roussean. Ia critica del gitusnamualismo nei "Discours", Catania, Bonnano, 1965. Como tal critica no supera el nivel alcanzado por R. Derathé: J.J. Rousseau et la philosophie politique de son temps, Paris, J. Vrin, 1970 (2e éd. mise au jour).

8. P. Casini: whantichiti e la ricerca della patria ideales, Rousseau selon Jean-Jacques. Coloquio di Roma, 5-6 mayo 1978, Florencia, Instituto della Enciclopedia Italiana e Université de Genève, $1979,87-96$.

9. Asume, matizándola, la tesis de J.L. Talmon: The Rise of totalitartan Democracy, Londres, Sacker and warburg, 1952.

10. Sobre esta tesis véase: J. Dehaussy: «Rousseau theoricien de la démocratien, Les Cahiers de la République, 50 (1962), 919-931; M. Imboden: Rousseau und die Demokratie, Tubingen, Morh, 1963; 1. Felscher: Rousseaus politische Philosophie. Zur Geschichte des demokratischen Freiheittsbegriffs, Berlin, Neuwied am $R$ und Luchterhand, $1960\left(2{ }^{a}\right.$ ed., 1968); J.L.L. Aranguren: *La vía democrática de Rousseats, en Ética y po- litica, Madrid, Guadarrama, 1968 (2." ed.) 137148; J. Mureau: Rousseau y la fundamentacion de la democracia (trad. de Juan del Agua), Madrid, Espasa-Calpe, 1977.

11. Annales de philosophie Politique, 5, Paris, P.U.F, 1965.

12. A. Sánchez Vázquez: "La filosofia de Rousseau y su influencia en Méxicon, en Presencia de Roussean. A los 250 años de su nacimiento y a los dos siglos de la aparición del Emilio y El Contrato social, México UNAM, 1962. A la doble conmemoración está también dedicado: $J . J$. Rousseau et son oetwre, Problemes et recherches, Collom que de París (16-20 octubre, 1962), París, C. Klincksieck, 1964, que estudia El Contrato social y otras tres obras. De caracter global es la recopilación de textos: Presencia de Rousseau, Buenos Aires, Nueva Visión, 1972, con estudios tan importantes como los de Lévi-Strauss, Derrida, Blanchot, Althusser.

13. O. Vossler: Rousseau Freitslehre, Göttingen, Vandenhoeck y Ruprecht, 1963.

14. I. Fetscher: Rousseaus politische Philosophie. Zur Geschichte des demokratischen Freihetsbegriffs, Berlin, Neuwied am R. un Luchterhand, 1960 (2." ed., 1968) (trad. it., Feltrinelli, 1972 y 1977); 1a 2." ed. incluye el Apếndice III ( do de naturaleza y la imagen del hombre en Hobbes, Pufendorf, Cumberland y Rousseaun) y la reelaboración del cap. 5 (*Rousseau y la Revolución francesam).

15. B. Baczko: Rousseau; samaność i wspolnota, Varsovia, 1964 (tr. fr.: Rousseau: solitude et communaute, Paris, La Haya, Mouton, 1974); el autor presento también una comunicación al coloquio de Ginebra (1962) con el título: aRousseau et lalienation socials, Annates J.J. Rousseau, t. XXXV (1959-1962), 223-237.

16. R. D. Masters: The political Philosophy of Rousseau, Princeton, Princeton University Press, 1968; «La science politique chez Rousseau ", Critique, 21 (1965), 965-973. Cf. A. Cobban: Rousseau and the modem State, new and rev. ed., Londres, G. Allen and Unwin, 1968.

17. G. Della Volpe: Rousseau e Marx, Roma, c. Riuniti, 1964 (tr. esp. Barcelona, Martínez Roca, 1969). L. Colletti: "Rousseau critico della "società civile" ", en su obra Ideologia e società, Bari, Laterza, 1969 (ir. esp. en Barcelona, Fontanella, 1975). B. Baczko: Rousseat: solitude er communaute, París, La Haya, Mouton, 1974. J.L. Lecercle: J.J. Rousseau, modernité d'un classique, París, Larousse, 1973; Du Contrat social, ed. de J.L. Lecercle, París, Éd. sociales, 1971.

18. J.N. Shklar: Man and Citizens. A Study of Rousseau's social Theory, Cambridge, Cambridge 
University Press, 1969. M. Berman: The Politios of Aathenticity. Radical Individualism and the emergence of modem Society, Nueva York, Atheneum, 1970. J. Charvet: The social Problem in the Philo. sophy of Rousseau, Cambridge, Cambridge University Press, 1974.

19. Madrid, Taurus, 1983, p. 42: I.J. Rousseau. La transparence et l'obstacte. Suivi de Sept essais sur Rousseau, Paris, Gallimard, 1971 (1. ${ }^{*}$ ed., 1957); los siete ensayos están incorporados en la edicion en castellano.

20. Rousseau after 200 years, ed, by R.A: Leigh, Cambridge, Cambridge University Press, 1982.

21. J.G. Merquior: Rousseat and Weber. Two Studies in the Theory of Legitimacy, Londres, Boston, Routledge y Keagan P., 1980.

22. Cfr. el excelente ensayo de J.L. Rodriguez Garcia: La mitada de Satumo. (Pensar la Revolución 1789-1850), Madrid, Ed. Revolución, 1990.

23. F. Furet et R. Halevi: Introducción a Orateturs de la Revolution française. $l$. Les Constituantes, ed. de F. Furet et R. Halévi, Gallimard (Pléiade), 1989, p. XCIV.

24. D. Mornet: Origines Intellectuelles de la Revolution frangaise, Paris, 1933.

25. R. Barny: "La Revolución y la Ihustración", en M. J. Villaverde (comp.). Alcance y legado de la Revolución francesa. Madrid, Ed. Pablo Iglesias, 1989, p. 69. Cfr. del mismo autor: Prelude ideologique à la Révolution française: le rousseaunisme avant 1789, Paris, Université de Besançon, 1985.

26. Dix-huttieme stècle, 21 (1989), dedicado a la influencia de Montesquieu en la Revolución francesa. B. Groetheysen (Philosophie de la RevoIution francaise, Gallimard, 1956; rémpr. 1982) estudia, además de la influencia de Montesquieu, las de Voltaire y Rousseau.

27. A. Soboul: «J.J. Rousseau et le Jacobinisme", Etudes sur le "Contrat social", cit. en Nota 6; Las sans-culottes, Movimiento popular y gobierro revolucionario (trad. de M. Ruipercz), Madrid. Alianza, 1987 (Paris, 1964); La Revolución francesa: principios ideológicos y protagonistas colectivos (trad de P. Bordonaba), Barcelona, Crítica, 1987 (Paris, 1982).

28. I. Starobinski: «Rotusseau y la Revolución francesa", en 1789 , los emblemas de la razón, Madrid, Taurus, 1988, pp. 149-150 (Instituto Editoriale Italiano, Milán, 1973; Flammarion, Paris 1979). Cfr. J. McDonald: Rousseau and the french Revolution, 1762-1791, Londres, Atlone Press, 1965 (I. Fetscher revisa el cap. 5 sobre Rousseau y la Revolución francesa inspirándose en este libro). L. Sozzi: "Interpretations de Rousseau perdant la Révolution*, Studies on Voltaire and XVIIth Century, t. LXIV, 1968, 187-223.

29. Nueva Vision, Buenos Aires, 1972. Véase nota 11 .

30. El libro de Colletti contiene el importante ensayo: "Rousscau, critico de la "sociedad civil" pp. 207-277, además de *Mandeville, Rousseau y Smith", pp. 279-309. El de Moreau (Paris, P.U.F., 1973) es un estudio global del pensamienzo de Rousseau, en el que dedica los cinco últimos capítulos al problema polítioo. Sobre el excelente estudio de Starobinski, ver nota 18. Acerca del tibro de Della Volpe (Roma, Editori Riuniti, 1964), ver notas 17 y 47 .

31. G. Sartori: Teoria de la democracia (trad, de S. Sánchez González), Madrid, Alianza, 1988, 2 vols. (Chatham Haouse Publishers, 1987). R. Grinsley: La fllosoffa de Roussean (trad. de J. Rubio), Madrid, Alianza, 1977 (Oxford University Press, 1973). G. Broethurysen: J.I. Rousseau (trad. de A. Garzón del Camino), Mexico, FCE, 1985 (Paris, Gallimard, 1949).

32. La edición de Alianza contiene prólogo y notas de M. Amriño. Escritos de combate no traduce una obra concreta de Rousseau; bajo este título se designa los dos Discursos y las respectivas controversias, el Contrato y la Carta a $\mathrm{C}$. de Beaunoni; la trad. y notas es de $S$. Masó y la introducción de $G$. Benrekassa.

33. J.J. Rousseau: Disc. Econ pol, ed, de E. Candela. Proyecto y Consideraciones, ed. de A. Hermosa Andujar. Disc. orig. desig., ed. de A. Pintor Ramos. El Contrato social, ed de M.J. Villaverde. De la última obra se han becho 13 ediciones en castellano en las tres úlimas décadas.

34. J.J. Rousseau: Escritos sobre la paz y la guerra, (pról. de A. Truyol y Serra), Centro de Estudios Constitucionales, 1982; Emilio o De la Educación (pról. de M.C. Iglesias), Madrid, Edaf, 1985.

35. M.J. Villaverde: Rousseau y el pensamiento de las Luces, Madrid, Tecnos, 1987; Rousseau. El consenadarismo de th mito, Madrid, Universidad Complutense, 1986.

36. J. López Hernánder: La ley det corazón (ton estudio sobre J.J. Roussean), Murcia, Publicaciones Universidad de Murcia, 1989; "El problema del derecho natural en J.J. Rousseau*, Antiario de Filosofia del Derecho, Madrid (1987), 539-562; «J.I. Rousseau, el fracaso de la razion polítican, Anoles de Filosofia, vol. I (1983), 157-167.

37. F.J. Caballero Harriet; Naturaleza y Derecho en J.J. Rousseau. Bilbao, Universidad del País Vasco, 1986. A. Ginzo: La Ilustracion francesa. Entre Voltaine y Rousseau, Madrid, Cincel, 1985. G. Dalmaso: La politica de to imaginario: Rousseau-Sade, Madrid, Encuentro, 1983.

38. E. Bello: "Lectura kantiana del Contrato social», en Kant después de Kant, ed de J. Muguerza y R. Rodriguez Aramayo, Madrid, Tecnos, 1989, 153-173. J. Rubio Carracedo: El influjo de Rousseau en la filosofía práctica de Kant *, en Esplendor y miseria de la ética kantiana, ed de E. Guisán, Barcelona, Anthropos 1988, 29-74. 
A. Hermosa Andujar: «El camino de Rousseau. De la democracia directa a la democracia representativa", Rev. de Estudios polticos, 50 (1986), 101-141. M. Bonachela: "Comentarios sobre el principio de la separación de poderes en J.J. Rousseau», Rew. Estudios politicos, 28 (1982), 75 123.

39. J. de Maistre (1796): Considerations sur la France, éd. de J.L. Darcel, Ginebra, 1980 (trad. cast. en Tecnos, 1990, con pról. de A. Truyol y Serra). Chateaubriand, Essai sur les révolutions (1797).

40. L.G. Crocker (Cleveland, Press of Case W. Reserve University, 1968) ha publicado, ademas, una biografía (Jean-Jacques Rousseau, Nueva York, Macmillan, 1968-1973,2 vols.) y diferentes articulos sobre la misma tesis (eRousseau et la voie du totalitarismes, en La phtiosophie politique de J.J. Rousseau, París, P.U.F., 1965); se inspira en J.L. Talmon; The rise of totalitarian Democracy, Nueva York, Praeger, 1960 (1st ed., Boston, 1952).

41. B. Constant: De la libertad de los antiguos comparada con la libertad de los modernos: en Del espiritu de conquista, Madrid, Tecnos, 1988, 63-93; Oezures, éd. de A. Roulin, Paris, Gallimard, 1957; S. Goyard-Fabre: "L'idec de souverainete du peuple et le "liberalisme pur" de B. Constant", Rev, de Met. et Mor, 81 (1976), 289. 327. A. de Tocqueville: El Antiguo régimen y la Revolución, Madrid, Alianza, 1982, 2 vols.; La democracia en America, Madrid, Alianza, 1980, 2 vols.

42. L.J. Halle: The ideological Imagination. Ideological Conflict in our Tine and its Roots in Hobbes, Rotisseat and Marx, Londres, Chato and Windus, 1972 (piensa Halle que Rousseau potencia el totalitarismo que viene de Hobbes y llega hasta el marxismo, contrario al ideal liberal). M.J. Villaverde: v. nota 34. E. Cassirer: Rousseau, Kant, Goethe. Two Essays, Princeton, Princelon University Press, 1970 (1st ed., 1945). P. Pasqualucci (Rousseau e Kant, Milán, A Giuflrè, 19741976, 2 vols.) critica la intepretación neokantiana, sosteniendo que Rousscau es el primer eslabón de la cadena que lleva a Hegel y a Marx. A. Levine (The Politics of Autonomy, A kantian Reading of Rousseau's a Social Contract", Amherst, University of Mass. Press, 1976), en lugar de hacer un estudio de la relacion Rousseau/Kant, se limita a divulgar los análisis althusserianos de El Contrato social; sólo se ocupa de Kant al final del libro, para concluir diciendo que Rousseau prefigura a Kant, que es la tesis de Cassirer. P.C. MayerTasch: Autonomie und Attoritat. Roussedu in den Spuren von Hobbes?, Berlin, Luchterhand, 1968. H. Schmidt: Seinserkenntnis und Staatsdenker. Der Subiekts - und Erkennisbergriff von Hobbes, Locke und Rousseau als Grundlage des Rechtes tha der Geschichte, Tübingen, M. Miemeyer, 1965. D. Cameron: The social Thought of Rousseau and Burke: a comparative Study, Londres. Toronto, Weidenfeld and N., 1973. K, Martin: French liberal Thought in the eighteenth Centary: a Study of political lateas from Bayle to Condorcet, ed. by J.P. Mayer, Nueva York, Harper and Row, 1963 (1st 1929).

43. J.W. Chapman: Rousseau, totaltarian or liberal?, Nueva York, Columbia University Press, 1956.

44. Cf. G. Buglé: "Rousseau et le socialisme», Rev. de Mét. et Mor, 3 (1912), 341.

45. L. Althusser- "Sur le "Contrat social" (Les Décalages). Limpense de J.J. Rousseau, Cahiers pour lAnalyse, 8, Paris, Le Graphe (1967). A. Levine (The Poitics of Autonomy, cit. en nota 42) divulga en lengua inglesa los analisis de Althusser.

46. A. Illuminati: $J . J$. Roussean e la fondazione dei valori borghesi, Milán, II Saggiatore, 1977; Jean-Jacques Rousseau, Florencia, La Nuova Italia, 1975. Dentro de esta línea descalificadora, $A$. Domenech solo subraya lo que califica de republicanismo "absolutista" de Rousseau" (De ta etica a la polttica. Barcelona, Critica, 1989 , p. 265).

47. G. Della Volpe: Roussean y Marx, Barcelona, Martínez Roca, $1969, \mathrm{p}, 15$.

48. L. Colletti: «Rousseau, crítico de la "sociedad civil"x, en Ideologia y sociedad. Barcelona. Fontanella, 1975, pp. 263-264.

49. B. Baczko: Roussean: solitude et communauté, París, La Haya, Mouton, $1974\left(1 .^{2}\right.$ ed. Varsovia, 1964). J.L. Lecercle: J.J. Rousseau, modernité d'un classique, París, Larousse, 1973, p. 244.

50. CF. E. Cassiter: «Das Problem J.J. Rousm seau», Archiv fur Geschichte der Philosophie, XLI (1932), 177-213 y $479-513$

51. E. Díaz: Estado de Derecho y sociedad democratica, Madrid, Taurus, 1981 (8.3 ed.), p. 29.

52. N. Bobbio: "La crisis de la democracia y la lección de los clásicos», Crisis de la democracia, Barcelona, Ariel, 1985, pp. 5-25; aDemocracja representativa y democracia directas, El futuro de la democracia, Mexico, FCE, 1986, pp. 32-50. Cfr. José M. Gonzalez: "Límites y aporias de la democracia representativa en $\mathrm{N}$. Bobbios, en Teortas de la democracta, ed. de J.M. Gonallez y F. Quesada, Barcelona, Anthropos, 1988, pp. 38-55.

53. L. Colletti: Ideologia y sociedad, cit., p. 266.

54. J. Rawls: A Theory of Justice, Oxford, Oxford University Press, 1972, p. 11 (trad. de M.D. Gonzảlez), México, FCE, 1978, p. 28. Cfr. A. Levine: «eyond Justice: Rousseau againts Rawlsi, Jounal of Chinese Philosophy, 4 (1977), 123-142; véase el libro de A. Levine sobre Rousseau en notas 42 y 45 .

55. N. Bobbio: "Contrato y contractualismo en el debate actuali, en El futuro de la democracia México, FCE, 1986, pp. 114-118. Como variantes 
del debate, véase F. Vallespin Oña: Nuevas teorias del contrato social: $J$. Rawls, $R$. Nozick y $J$. Buchanor, Madrid, Alianza, 1985; S, Goyard-Fabre: Linterninable querelle du contrat social, Ottawa, Editions de l'Université d'Ottawa, 1983.

56. N. Bobbio: Estado, gobtemo, sociedad (trad. de L. Sánchez Garcia), Barcelona, Plaza y Janés, 1987, p. 163. Cfr. J.M. Gonzalez y F. Quesada (coords.): Teorfas de la democractia, Barcelona, Anthropos, 1988.

57. E. Díaz: Estado de Derecho y sociedad democrdica, Madrid, Taurus, 1981 ( $8^{\mathrm{a}}$ ed.), pp. 38. 39; De la maldad estatal y la soberamia popular, Madrid, Debate, 1984, pp. 56-57; A. Maestre: «Re- nexión para una ética en democracia: discurso ético y utopias, en Teorias de la democracia, cit., pp. 79-107; J. Muguerza: "Etica y comunicación. (Una discusión del pensamiento ético-político de Jürgen Habermas) , ibid., pp. 108-171; J. Muguerza et al. El fundamento de los derechos hamanos, Madrid, Debate, 1989.

58. Londres, Boston, Routledge and Kegan Panl, 1980. Cfr. M.E. Perkins: wLiberty and the Concept of Legitimacy in the "Discours sur linc. galité" ". Actes du IIIe Congrès Intemational sur le "Siecle des Lumières", Nancy, 1971 (ed. Banbury, 1972).

\title{
Obediencia al Derecho: revisión de una polémica
}

\author{
JUAN RAMÓN DE PÁRAMO \\ Universidad Complutense
}

Desde la publicación en 1979 del arlículo del profesor González Vicén sobre la obediencia al Derecho se ha suscitado una discusión sobre su contenido en la que han venido participando algunos miembros del gremio institucionalizado "Filosofia del Derecho, moral y polítican, unidos, esta vez sí, por un debate ético común (González Vicén 1979, 1985 y 1989; Atienza, 1983; Díaz, 1984, 1985 y 1988; Muguerza, 1986, 1987 y 1989; Fernández, 1987 y 1989; Cortina, 1987 y 1989; Guisán, 1988). Si bien parte de los argumentos en discusión han versado sobre los rasgos descriptivos del Derecho -el Derecho como institución social que disciplina y organiza la fuerza en una comunidad-, el problema de fondo ha consistido en analizar la vinculatoriedad jurídica, es decir, los diversos tipos de razones - si es que existenque ofrecen las normas jurídicas para justificar acciones o decisiones. En particular, si las normas jurídicas ofre- cen razones morales para justificar su obediencia; si existe una obligación moral de obedecer al Derecho. A veces este problema no se ha enunciado del mismo modo en la discusión, lo que ha producido respuestas diferentes.

González. Vicén (1979) partía en su argumentación de la crítica de una versión del positivismo legal -que, por cierto, no creo ni dominante ni relevante en la versión actual del positivismo jurídico- que sostiene que todo Derecho producido correctamente y revestido de validez formal obliga con independencia de su contenido. La vinculatoriedad jurídica significa aquí obligatoriedad del Derecho tan sólo por razón de su validez formal. A partir de la exposición crítica de esta tesis, González Vicén examinaba con detenimiento algunas respuestas históricas a tal cuestión: la teoría del derecho del más fuerte, del reconocimiento, del iusnaturalismo y de la seguridad jurídica; se ponía de manifiesto sus con- 